\title{
Evaluación del seguimiento radiográfico para desplazamiento de cadera en pacientes con parálisis cerebral en el Centro Hospitalario Pereira Rossell
}

Dres. Ignacio Rehbein*, Viviana Teske ${ }^{\dagger}$, Johan von Heideken‡

\section{Resumen}

Introducción: la luxación de cadera es una complicación severa en pacientes con parálisis cerebral (PC), sobre todo en pacientes incluidos en el sistema de clasificación de la función motora gruesa (GMFCS, por su sigla en inglés) III-V. Para su identificación son necesarias radiografías de pelvis.

Objetivos: evaluar el seguimiento radiográfico en estos pacientes y la detección precoz de esta complicación en nuestro hospital.

Material y método: se revisaron historias clínicas y radiografías de 17 pacientes GMFCS III-V, entre 2 y 8 años de edad al momento de la radiografía de pelvis índice, midiendo el porcentaje de migración (PM) de cadera de acuerdo al índice de Reimer, el ángulo cérvico-diafisiario y calculando el CPUP Score de cada cadera. Evaluamos el control radiográfico al año 0 posteriormente a esa fecha, y de no haber sido así, se citaría a los pacientes a control radiológico para detectar las caderas con riesgo migratorio elevado.

Resultados: de los 17 pacientes evaluados, 3 (18\%) tuvieron una nueva radiografía de pelvis al año; 6 (35\%) pacientes la tuvieron posteriormente al año, y antes de la fecha de control designada, 7 (41\%) pacientes nunca fueron controlados, citándose para nueva radiografía en 2018. Un paciente (6\%) se perdió en el seguimiento. Un paciente presentó una cadera con riesgo alto (CPUP Score 50\%-60\%), el resto tuvo PM dentro de rangos normales.

Conclusiones: pocos pacientes con PC GMFCS III-V tuvieron un seguimiento radiográfico anual. Los monitoreados posteriormente no mostraron progresión de esta condición. El resultado de este estudio y la literatura respaldan la introducción de un programa de vigilancia en nuestro hospital.

Palabras clave: Parálisis cerebral Luxación de la cadera

Key words: Cerebral palsy Radiografía Hip dislocation

Niño Radiography

Child

\footnotetext{
* Médico residente de Ortopedia y Traumatología. Clínica de Traumatología y Ortopedia Pediátrica, Facultad de Medicina, Universidad de la República. Montevideo, Uruguay

† Profesora adjunta Clínica de Traumatología y Ortopedia Pediátrica, Facultad de Medicina, Universidad de la República. Montevideo, Uruguay. ‡ Departamento de Salud del Niño y de la Mujer, Instituto Karolinska, Estocolmo, Suecia.

Estudio llevado a cabo en la Clínica de Traumatología y Ortopedia Pediátrica, Centro Hospitalario Pereira Rossell, Facultad de Medicina, Universidad de la República. Montevideo, Uruguay

Los autores declaran no tener conflictos de interés.

Este estudio fue aprobado por el Comité de Ética en Investigación del CHPR.

Correspondencia Dr. Ignacio Rehbein. Centro Hospitalario Pereira Rossell. Bulevar Artigas 1550, CP 11600. Montevideo, Uruguay. Correo elec-

trónico: rehbeinresidente@gmail.com

Recibido: 14/4/19

Aprobado: 9/9/19
} 


\section{Introducción}

La parálisis cerebral (PC) es la causa más común de discapacidad motora en la infancia. La prevalencia de PC varía de 1,5 a 3 por 1.000 nacidos vivos ${ }^{(1)}$. Consiste en una encefalopatía no progresiva que deriva en aumento del tono muscular, paresia, contracturas musculares, inestabilidad articular y deformidades musculoesqueléticas, todo lo cual conduce a limitaciones de la actividad. El desplazamiento de cadera en niños con parálisis cerebral es frecuente ${ }^{(1)}$, con un porcentaje de migración (PM) entre 33\% y 100\%, siendo indoloro en etapas iniciales para el paciente, mientras que en etapas avanzadas, cuando la cadera se encuentra luxada (PM 100\%), puede causar dolor, contracturas severas, deformidad ráfaga de viento, limitación funcional y enfermedad degenerativa de la cadera que compromete el posicionamiento, sedestación, bipedestación, habilidad para caminar y calidad de vida, en general, tanto para el paciente como para el cuidador, pudiendo convertirse en un problema serio y de difícil solución. El riesgo de progresión a luxación de cadera es de $15 \%$ a $20 \%$ en la población total de niños con $\mathrm{PC}^{(2)}$. Es por esto que su detección a temprana edad es fundamental. Se han establecido factores de riesgo para el desplazamiento de cadera. Según Pruszczynski y colaboradores ${ }^{(3)}$, los factores de riesgo a tener en cuenta son el nivel de GMFCS, a mayor nivel, mayor probabilidad de migración; la edad, registrándose un pico entre los 3 y 5 años; los desórdenes de tono muscular y movimiento, mayor en diplejía espástica y distonía, y tasa de aumento del desplazamiento de cadera, evaluada comúnmente por PM o índice de Reimers. Se considera un índice de Reimers $<33 \%$ como normal, entre $33 \%$ y $40 \%$ como cadera en riesgo, $>40 \%$ como cadera subluxada y $100 \%$ cuando la cadera está luxada ${ }^{(4)}$. Los pacientes con PM $>40 \%$ tienen un alto riesgo de mayor desplazamiento, por lo que la cirugía está indicada en esos casos. En los niños con un PM entre $33 \%-40 \%$, se deben evaluar otros parámetros para decidir el manejo, incluida la condición clínica, el subtipo de PC y la edad ${ }^{(5)}$. Muchos pacientes con PC también se presentan con coxa valga, con una cabeza femoral con valgo aumentado en relación con el cuello femoral. Estas deformidades se presentan en combinación y se pueden medir con el ángulo cérvico-diafisiario (ACD), ángulo que se ha demostrado que aumenta en los niños con PC y que ha demostrado ser un factor de riesgo válido para el desplazamiento de la cadera en esta cohorte de pacientes ${ }^{(6)}$. Existen formas de tratamiento conservador para prevenir la migración de cadera, entre las que destacan terapia física, férulas (aparatos ortopédicos), toxina botulínica, marcos de abducción y sillas moldeadas especiales, con efectividad limitada, logrando solo retrasar la aparición de esta complicación ${ }^{(7)}$. En cuanto al tratamiento quirúrgico, se puede dividir en tratamiento preventivo de la progresión de la migración de cadera con cirugía de partes blandas (liberación aductor, psoas e isquiotibial), el tratamiento reconstructivo; con osteotomía varizante desrrotadora con o sin osteotomía periacetabular de pelvis, y tratamiento de salvataje, con la resección del tercio proximal de fémur ${ }^{(7)}$. La literatura actual avala fuertemente la efectividad de un programa de seguimiento específicamente diseñado con radiografías anuales de pelvis para identificar pacientes con desplazamiento de cadera. Varias naciones, entre ellos los países escandinavos y Australia, poseen programas de vigilancia para el screening de cadera basados en la población ${ }^{(8-10)}$. Según nuestro conocimiento, no existen programas de vigilancia de PC en América Central ni en Sudamérica.

Hermanson y colaboradores crearon en 2015 el CPUP Score (Programa de seguimiento para la parálisis cerebral, Uppföljningsprogram för cerebral pares, por su sigla en sueco). Este score tiene la capacidad de determinar el riesgo de desplazamiento de cadera ( $\mathrm{PM}>40 \%)$ dentro de cinco años en niños con PC, en GMFCS III-V, siendo de alto o bajo riesgo. CPUP Score implica una detección temprana de caderas en riesgo a través de un seguimiento clínico y radiográfico sistemático. Este score usa el PM, el ACD, la edad del paciente y el tipo de PC para ayudar a determinar el mejor tratamiento para el paciente, brindando la oportunidad de tratar a más pacientes con cirugía menos agresiva ${ }^{(5)}$.

En el año 2015, en el Centro Hospitalario Pereira Rossell (CHPR), lugar de nuestro estudio, se creó una nueva clínica de PC entre cirujanos ortopédicos, fisiatras y psicólogos, brindando atención ortopédica en una base más especializada. Esta clínica le da al paciente la posibilidad de un chequeo más exhaustivo, junto con una atención más personalizada con respecto al espectro de comorbilidades en los pacientes con PC, incluida la luxación de cadera. Es por esto que en el presente estudio queremos evaluar la práctica ortopédica actual con respecto a la vigilancia de la luxación de cadera en nuestra institución, con el fin de proporcionar una base para la mejora en la atención ortopédica. Según el protocolo CHPR, los niños con GMFCS III-V deben ser controlados clínica y radiológicamente una vez al año, desde los 2 hasta los 8 años. Posteriormente a esa edad, el intervalo de tiempo entre controles es determinado individualmente basado en el resultado del examen previo, en concordancia con las prácticas internacionales ${ }^{(11)}$.

Este es un estudio observacional que describe la práctica clínica en nuestra institución respecto al desplazamiento de cadera y nuestros objetivos principales son 


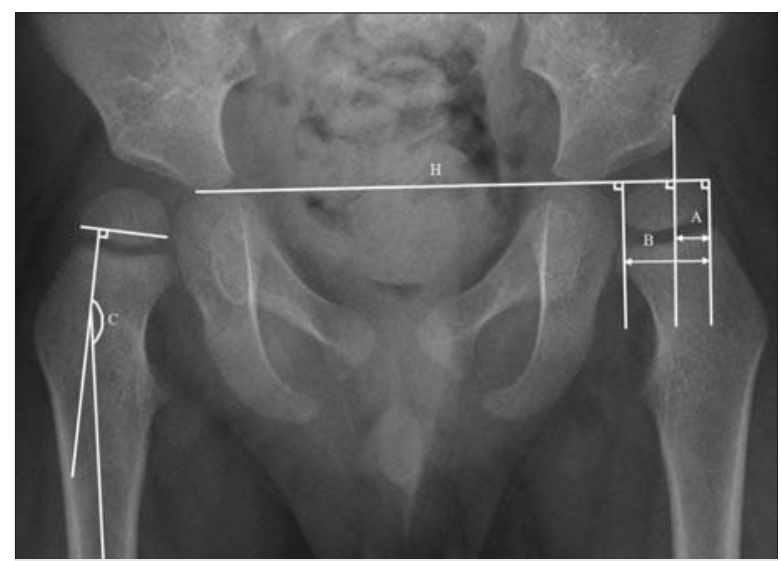

Figura 1. Radiografía frente $(F)$ de pelvis. Cadera derecha: ángulo cérvico-diafisiario (C) midiendo el ángulo interno entre una línea perpendicular a la epífisis proximal de fémur y una línea a través de la diáfisis femoral. Cadera izquierda: porcentaje de migración (PM) medido por una línea de Hilgenreiner $(H)$ y tres líneas perpendiculares, utilizando $A / B x$ 100\%. Imagen tomada de Van der List, et al. J Child Orthop (2015) 9:129-35.

determinar si a estos pacientes se les realizó una nueva radiografía al año, o según necesidad, y como objetivo secundario obtener el valor CPUP Score, PM y ACD, y establecer si había alguna cadera con alto riesgo de progresión del desplazamiento. Nuestra hipótesis es que los pacientes con PC GMFCS III-V deberían ser controlados con radiografía de pelvis en forma anual.

\section{Pacientes y método}

En el año 2016 se revisó la base de datos electrónica del block quirúrgico del CHPR, evaluando todos las historias clínicas y radiografías de los pacientes registrados con diagnóstico de PC que fueron operados con cirugía ortopédica. De estos pacientes, se obtuvieron datos demográficos (edad, sexo, fecha de nacimiento), nivel de GMFCS, subtipo de parálisis cerebral (bilateral espástica, unilateral espástica, disquinesia, atáxica, mixta y no clasificable), fecha y edad del paciente al momento de la última radiografía de pelvis registrada. El protocolo fue aprobado por el Comité de Ética de la institución.

En el presente estudio se utilizará, de la misma base de datos obtenida previamente, como análisis secundario de la cohorte antes mencionada, a los pacientes con los siguientes criterios de inclusión:

- Niños entre 2 y 8 años de edad al momento de la última radiografía de pelvis revisada en el estudio inicial.

- Diagnóstico de PC con nivel GMFCS III-V.
- PM de acuerdo al índice de Reimers $<40 \%$.

- Que no hayan recibido cirugía ósea de la cadera.

- Que su última radiografía de pelvis se haya realizado durante el año 2015 o posteriormente.

No se incluyeron pacientes menores de 2 años dado que el diagnóstico de PC usualmente se realiza sobre esta edad ${ }^{(1)}$, y no se incluyeron pacientes mayores de 8 años debido a la baja probabilidad de desplazamiento de cadera que se presenta en esta edad ${ }^{(15)}$. No se incluyeron pacientes con GMFCS menor a III, dada la baja tasa de progresión en el desplazamiento de cadera que presentan estos pacientes ${ }^{(3)}$. Pacientes con PM $>40 \%$ generalmente requieren cirugía ósea de entrada ${ }^{(16)}$, por lo que dada su alteración anatómica (especialmente ACD) resultante, se excluyeron pacientes en quienes se realizó cirugía ósea de pelvis o cadera, o ambas.

Utilizamos la última radiografía tomada de los años 2015-2016 como radiografía índice y calculamos el tiempo (meses) hasta la nueva radiografía de pelvis de control. En caso de que el paciente no tuviese nueva radiografía de control, se le contactaría y citaría para realizarse una nueva.

En la radiografía índice se establecieron el porcentaje de migración según el método de Reimers y el ACD, medidos manualmente en la última radiografía de pelvis encontrada, tomada con rotación interna de cadera y CPUP Score utilizando la aplicación oficial de su sitio $w^{2} b^{(17)}$ y tomando como parámetros el nivel de GMFCS, edad, valor de PM y ACD.

El ACD se obtiene midiendo el ángulo medial entre una línea perpendicular a la epífisis femoral proximal y una línea a través del medio de la diáfisis femoral, de acuerdo a Southwick ${ }^{(18)}$. El porcentaje de migración de la cabeza femoral se midió según el método de Reimers $^{(4)}$ (figura 1). Dado que la confiabilidad inter e intraobservador es excelente para el ACD con un coeficiente de correlación intraclase (CCI) de 0,92 (95\% intervalo de confianza [IC] y un CCI 0,99 (95\% IC $0,98-0,99)^{(19)}$ y la confiabilidad inter e intraobservador del PM es alta (CCI $=0,93$; $95 \%$ IC $0,91-0,95)^{(20)}$, la medición fue realizada por un único examinador ${ }^{(21)}$. En relación con el cálculo de CPUP Score ${ }^{(17)}$, este se realizó a través de la aplicación para dispositivos móviles, y se introdujeron manualmente los datos solicitados (GMFCS, edad, valor de PM y ACD) para cada cadera evaluada, arrojando como resultado el riesgo de progresión de migración expresado en porcentaje. Este score posee una alta habilidad predictiva para diferenciar entre riesgo alto y bajo (área bajo la curva de características del operador receptor $=0,87$ ).

De acuerdo al flujograma de pacientes (figura 2), de los 227 pacientes evaluados en la base de datos inicial, 


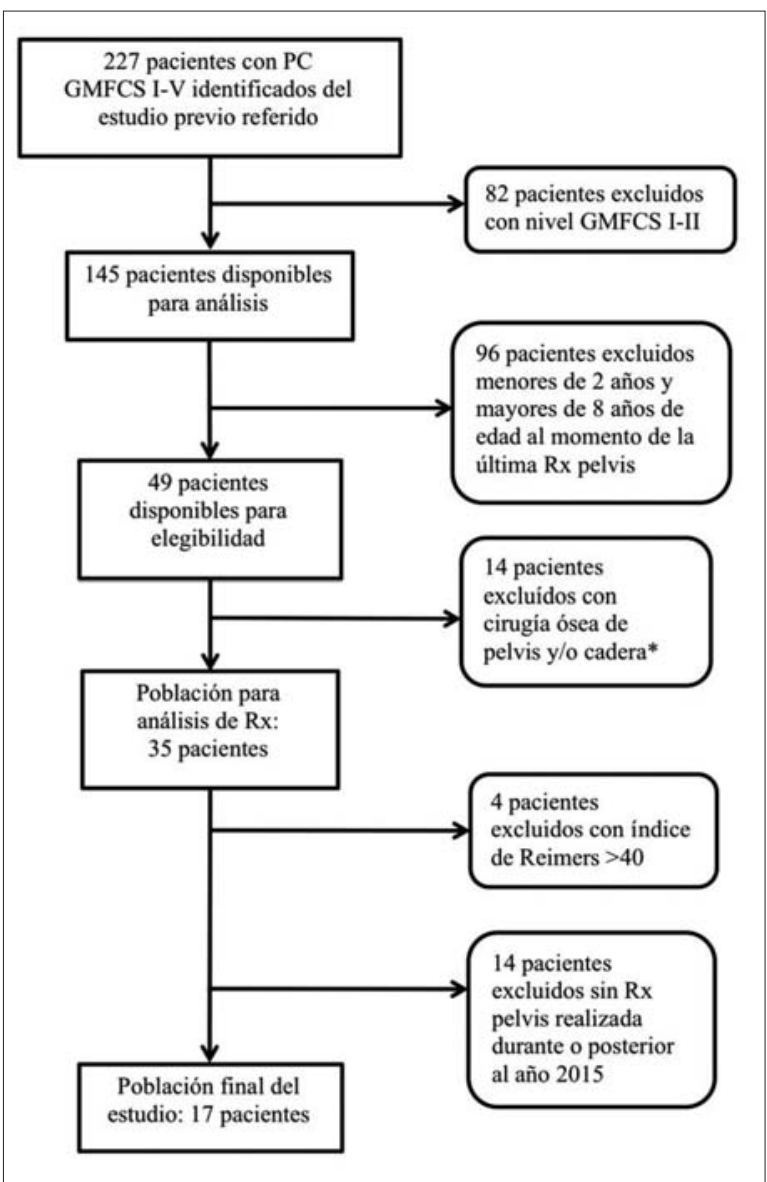

Figura 2. Flujograma de selección de pacientes. * Cirugía ósea en pelvis/cadera alteraría la anatomía de dicho segmento, con la consiguiente variación en PM y ACD.

GMFCS: Gross Motor Functional Classification System

17 cumplieron con los criterios de inclusión. Se realizaron mediciones de ACD y PM en ambas caderas, arrojando un total de 33 caderas evaluadas. A los dos años transcurridos, se volvieron a revisar las historias clínicas de los 17 pacientes seleccionados, buscando si se les había realizado una nueva radiografía de pelvis, y de estas nuevas radiografías tomadas, volver a medir PM para evaluar la progresión de dichos parámetros.

De no haberse realizado una nueva radiografía, se citaría a los pacientes en agosto de 2018 para su realización y posterior medición y valoración de la progresión de PM, si es que la hubo.

\section{Análisis estadístico}

Análisis descriptivo, como conteos, porcentajes y promedios con rango fueron utilizados para caracterizar la muestra. El tiempo entre la radiografía de pelvis índice y la radiografía de seguimiento se estratificó en

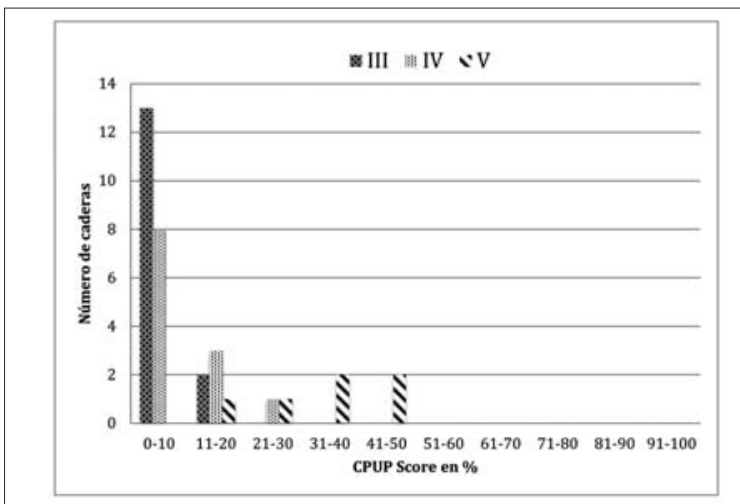

Figura 3. Número de caderas por GMFCS y CPUP Score, obtenido de radiografías índice (2015-2016). GMFCS: Gross Motor Functional Classification System; CPUP Score: porcentaje de riesgo de desplazamiento de cadera (PM $>40 \%$ ) dentro de cinco años.

menos de un año y más de un año. El CPUP Score medido en la radiografía índice se comparó entre los niveles de GMFCS.

\section{Resultados}

De la cohorte inicial de 227 pacientes, 17 cumplieron con los requisitos de inclusión (12 niños, 5 niñas). El promedio de edad al momento de la última radiografía registrada fue de 6 años (4-8). En la tabla 1 se detalla la información demográfica del grupo de estudio. La distribución según GMFCS fue de 8 pacientes nivel III, 6 pacientes nivel IV y 3 pacientes nivel V. Todos los pacientes se clasificaron según subtipo de $\mathrm{PC}$, la distribución fue: bilateral espástico con 16 pacientes, unilateral espástico con un paciente. No se registraron pacientes con disquinesia, ataxia, mixta y no clasificable. El total de caderas a evaluar fue de 33 . El promedio de PM de las caderas derechas fue de $21 \%(10 \%-38 \%)$ y el de caderas izquierdas fue de $23 \%$ (10\%-34\%). El ACD promedio fue en cadera derecha $160^{\circ}\left(149^{\circ}-171^{\circ}\right)$ y en cadera izquierda $162^{\circ}\left(148^{\circ}-178^{\circ}\right)$. La distribución de caderas, según GMFCS y CPUP Score, se muestra en la figura 3. En los pacientes GMFCS III, 13 caderas se encontraron en el rango de $0 \%-10 \%$ y dos caderas en $11 \%-20 \%$ de riesgo de desplazamiento de cadera en cinco años. De los pacientes GMFCS nivel IV, ocho caderas en rango $0 \%-10 \%$, tres caderas en $11 \%-20 \%$ y una cadera en $21 \%-30 \%$. El riesgo más alto de migración de cadera, de acuerdo al CPUP Score, se encontró en el grupo GMFCS nivel $\mathrm{V}$, con una cadera en rango $11 \%-20 \%$, una cadera en $21 \%-30 \%$, dos caderas en $31 \%-40 \%$ y dos caderas $41 \%-50 \%$. 


\begin{tabular}{|c|c|}
\hline Variable & $n$ (rango) \\
\hline Número total de pacientes & 17 \\
\hline $\begin{array}{l}\text { Promedio de edad al momento de la última radiografía } \\
\text { de pelvis, años }\end{array}$ & $6(4-8)$ \\
\hline \multicolumn{2}{|l|}{ Distribución por GMFCS } \\
\hline III & $8(47 \%)$ \\
\hline IV & $6(35 \%)$ \\
\hline V & $3(18 \%)$ \\
\hline \multicolumn{2}{|l|}{ Subtipo de PC } \\
\hline Espástico bilateral & $16(94 \%)$ \\
\hline Espástico unilateral & $1(6 \%)$ \\
\hline Disquinético & $0(0 \%)$ \\
\hline Atáxico & $0(0 \%)$ \\
\hline Mixta/No clasificable & $0(0 \%)$ \\
\hline \multicolumn{2}{|l|}{ Promedio PM } \\
\hline Cadera derecha* & $21(10-38)$ \\
\hline Cadera izquierda & $23(10-34)$ \\
\hline \multicolumn{2}{|l|}{ Promedio ACD } \\
\hline Cadera derecha* & $160(149-171)$ \\
\hline Cadera izquierda & $162(148-178)$ \\
\hline $\begin{array}{l}\text { * Excluida cadera sana de paciente unilateral espás } \\
\text { GMFCS: Gross Motor Functional Classification Sy } \\
\text { rálisis cerebral; PM: promedio de migración en por } \\
\text { ángulo cérvico-diafisiario en grados. }\end{array}$ & $\begin{array}{l}\text { ico. } \\
\text { stem; PC: pa- } \\
\text { centaje; ACD: }\end{array}$ \\
\hline
\end{tabular}

De los 17 pacientes seleccionados para nuestro estudio, solo a tres (18\%) se les realizó una nueva radiografía de pelvis durante el año siguiente a la evaluación inicial. En seis (35\%) pacientes se realizó una nueva radiografía después de más de 12 meses de evolución. Los pacientes restantes $(n=7,41 \%)$ nunca tuvieron una segunda radiografía de pelvis, siendo citados a nuevo control clínico y radiológico en agosto de 2018 . Un paciente (6\%) GMFCS IV que tenía su radiografía índice 35 meses atrás, no pudo acudir a control por encontrarse en mal estado de salud (tabla 2, figura 4).

A los pacientes GMFCS nivel III se les realizó una nueva radiografía de pelvis en promedio a los 23 meses (15-28) posteriores a la radiografía índice. A los GMFCS nivel IV se les realizó a los 27 meses (20-40) y a los pacientes GMFCS V se les realizó a los 25 (19-30).

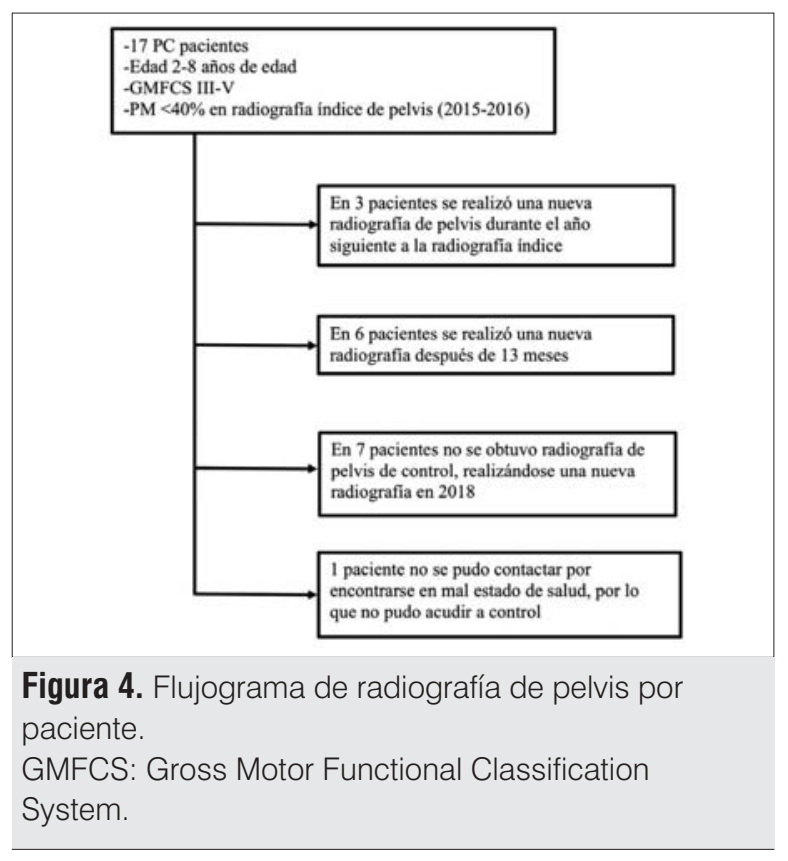

En las radiografías posteriores a las del índice en los pacientes GMFCS nivel III, el promedio de PM de cadera derecha aumentó de $20 \%$ a $23 \%$, el de cadera izquierda aumentó de 24\% a 26\%. En los GMFCS nivel IV, el promedio de PM disminuyó de $25 \%$ a $21 \%$ en ambas caderas. Y en los pacientes GMFCS V, el promedio de PM disminuyó de 26\% a 13\% en cadera derecha y de $20 \%$ a $15 \%$ en cadera izquierda.

\section{Discusión}

Este es un estudio observacional, retrospectivo, que evalúa nuestra práctica clínica en la detección de la migración de cadera en niños con PC GMFCS III-V, entre los 2 y 8 años de edad. No confirmamos nuestra hipótesis de que los niños tendrían sus radiografías de cadera anuales programadas. Nuestro principal hallazgo es que de los 17 pacientes evaluados, solo tres (18\%) se sometieron a control radiográfico según lo indicado.

Está más allá del alcance de este estudio evaluar las razones detrás de nuestros resultados. Solo podemos especular, pero probablemente se deba a varias razones: puede haber razones económicas por las cuales el paciente no pueda acudir al control, asociado a la complejidad de la condición del paciente que dificulta el transporte; los padres pueden no entender la importancia de la detección temprana de la migración de cadera, ya que en etapas iniciales no es dolorosa, y los padres pueden no priorizar una visita anual a control con ortopedista; puede haber una confusión generada en los padres respecto a la cantidad de indicaciones a seguir provenientes de los distintos profesionales de la salud involucrados en la atención de dichos pacientes. En algunos casos podría 
Tabla 2. Número de pacientes por GMFCS y promedio de meses posteriores a radiografía índice, hasta nuevo control con radiografía de pelvis.

\begin{tabular}{lcccccccc}
\hline GMFCS & \multicolumn{1}{c}{ III } & \multicolumn{1}{c}{ IV } & V & Total \\
\cline { 2 - 8 } & $N$ & Tiempo (rango) & $N$ & Tiempo (rango) & $N$ & Tiempo (rango) & N (\%) & Tiempo (rango) \\
\hline $\begin{array}{l}\text { Obtuvo radiografía } \\
\text { dentro de un año }\end{array}$ & 2 & $13(12-13)$ & & & 1 & 11 & $3(19)$ & $(11-13)$ \\
$\begin{array}{l}\text { Obtuvo radiografía } \\
\text { después de un año }\end{array}$ & 3 & $24(22-25)$ & 2 & $22(20-23)$ & 1 & 19 & $6(38)$ & $(19-25)$ \\
$\begin{array}{l}\text { Obtuvo radiografía en } \\
\text { control, agosto 2018 }\end{array}$ & 3 & $28(22-35)$ & 3 & $31(24-40)$ & 1 & 30 & $7(44)$ & $(22-40)$
\end{tabular}

GMFCS: Gross Motor Functional Classification System.

ser el equipo médico el que no ha comunicado la indicación e importancia de forma adecuada de controlar esta potencial complicación. Es importante evaluar si hay procedimientos administrativos en el hospital en cuanto a la notificación de la fecha de control a los padres.

Creemos que este estudio podría servir como base para analizar esta situación, ya que no solo se necesita un seguimiento regular de la migración de cadera, sino también un examen ortopédico general. Esto incluye examen de columna vertebral, evaluación de espasticidad y posibles contracturas articulares. Algunos de los problemas ortopédicos pueden ser de mayor urgencia que la migración de cadera.

Hasta donde sabemos, no hay estudios similares realizados en Uruguay. Los países con programas de vigilancia de cadera han demostrado una disminución significativa en la dislocación de cadera ${ }^{(8,22,23)}$. Después de la introducción del programa CPUP en Suecia, el número de niños con cadera dislocada disminuyó de $8 \%$ a $0,5 \%$ en la población de pacientes con PC. La razón de esto fue una combinación de detección temprana de migración progresiva de cadera, además de las rutinas estandarizadas para el tratamiento preventivo, incluida la rehabilitación después de la cirugía ${ }^{(2)}$. A nuestro entender, no hay un programa de vigilancia de la migración de cadera en pacientes con PC en Sudamérica. Un programa de seguimiento para la vigilancia continua y estandarizada de niños con PC en CHPR probablemente influiría en una mejora significativa de la atención ortopédica de nuestros niños.

Los objetivos secundarios de nuestro estudio fueron medir la PM y ACD de cada cadera en la radiografía de índice y determinar el CPUP Score para evaluar el riesgo de migración de la cadera. De acuerdo con este score, la mayoría de los pacientes (13/17) tenían un riesgo inferior a $30 \%$ para desarrollar un $\mathrm{PM}>40 \%$ en los próximos cinco años. Creemos que es fácil calcular el puntaje y puede ser útil en la práctica clínica para decidir un seguimiento y tratamiento más exhaustivo en niños con PC. Sin embargo, no conocemos ningún país, fuera de Suecia, que use el CPUP Score en la práctica clínica. Ninguna de las caderas evaluadas tuvo una progresión significativa de migración de cadera entre la radiografía inicial y el seguimiento. No obstante, no sabemos cuánto tiempo transcurrió antes del siguiente control para los pacientes que nunca tuvieron una segunda radiografía.

Dentro de las limitaciones de nuestro estudio retrospectivo, reconocemos que el número de pacientes es bajo y éstos fueron identificados a partir de una base de datos de pacientes con PC tratados quirúrgicamente. Como otra potencial limitación, encontramos que aproximadamente la mitad (52\%) de los pacientes en el presente trabajo tuvieron cirugía de liberación de aductores, la cual podría afectar la precisión del CPUP Score comparado con niños sin este tipo de cirugía. Sin embargo, este procedimiento de partes blandas no afecta el ACD, una de las variables utilizadas para calcular dicho score. Este sesgo de selección puede afectar el resultado y limitar la generalización. También existe la posibilidad de que los pacientes obtengan una radiografía de cadera en otra institución de salud.

Entre las fortalezas de nuestro estudio se encuentran el hecho de que, a nuestro entender, este es el primer trabajo que evalúa la práctica clínica y el seguimiento radiográfico de la migración de cadera en pacientes con PC en nuestro hospital y en Uruguay, y que de los 17 pacientes que cumplieron con los criterios de inclusión, se pudo evaluar clínica y radiológicamente a 16 , lo que representa un alto porcentaje (94\%) de la cohorte inicial, y un solo paciente $(6 \%)$ no pudo ser incluido en el análisis final. Además de la valoración previamente descripta, se 
le aplicó a cada paciente el CPUP Score, instrumento validado internacionalmente para determinar el riesgo de migración de cadera a cinco años, pudiendo además dar pie para una nueva evaluación en dicho plazo.

En conclusión, pocos pacientes con PC GMFCS III-V tuvieron seguimiento radiográfico anual. Los que fueron monitoreados posteriormente no mostraron progresión de esta condición. Si bien cada paciente con PC es único desde el punto de vista diagnóstico, terapéutico y de rehabilitación, la evidencia actual dicta que es mandatorio crear y aplicar un programa de vigilancia de esta complicación que tanto perjudica la calidad de vida del paciente, padres y cuidadores.

\section{Abstract}

Introduction: hip luxation is a severe complication in patients with cerebral palsy (CP), especially in gross motor functional classification system (GMFCS) III-V patients. Pelvic $\mathrm{x}$-rays are necessary to identify this condition.

Objectives: to assess the x-ray follow-up in these patients and the early detection of this complication in our hospital

Method: the medical records and x-rays of 17 GMFCS III-V patients who were between 2 and 8 years old at the time of index pelvic x-ray were reviewed, measurement of the hip migration percentage according to Reimer's index, the cervical-diaphyseal angle and calculation of the CPUP hip score for each hip. We assessed the follow-up x-ray a year later or later than that date, and in the even this had not happened, we would call the patients in for a control x-ray to identify the hips with an increase migration risk

Results: 3 (18\%) out of 17 patients assessed underwent a new pelvic $\mathrm{x}$-ray a year later. 6 patients $(35 \%)$ underwent one later than one year after and prior to the control visit planned, 7 (41\%) patients were never controlled and they were called for a new x-ray in 2018. One patient $(6 \%)$ was lost during follow-up. One patient had an increased risk hip (CPUP Score 50-60\%), the rest had normal migration percentages.

Conclusions: few patients with PC GMFCS III-V underwent annual follow-up. Those monitored did not evidence progression of this condition. This study and the relevant literature back up the introduction of a surveillance program in our hospital.

\section{Resumo}

Introdução: a luxação de quadril é uma complicação grave nos pacientes com paralisia cerebral (PC), principalmente nos pacientes "Gross motor funcional classificativo system (GMFCS) III-V”. Para sua identificação é necessário realizar radiografias de pélvis.
Objetivos: avaliar o seguimento radiográfico nesses pacientes e realizar a detecção precoce desta complicação no hospital.

Material e método: foram estudados os prontuários e radiografias de 17 pacientes GMFCS III-V, com idades entre 2 e 8 anos no momento da radiografia de pélvis índice, medindo a porcentagem de migração (PM) de quadril de acordo com o índice de Reimer, o ângulo cervico-diafisiario e calculando o CPUP Score de cada quadril. Foi feito o controle radiográfico um ano ou mais depois da data da realização da radiografia; quando não foi possível os pacientes foram convocados para a realização de controle radiológico para detectar os quadris com risco migratório elevado.

Resultados: dos 17 pacientes avaliados, 3 (18\%) realizaram uma nova radiografia de pélvis um ano depois da primeira, 6 (35\%) pacientes depois de um ano mas antes de ser convocados para controle, 7 (41\%) pacientes nunca foram controlados, sendo convocados para uma nova radiografia em 2018 . Um paciente $(6 \%)$ foi perdido. Um paciente apresentou um quadril com risco alto (CPUP Score 50-60\%), os demais tinham PM dentro de intervalos normais.

Conclusões: poucos pacientes com PC GMFCS III-V tiveram um seguimento radiográfico anual. Os que foram monitorados posteriormente não mostraram progressão desta condição. O resultado deste estudo e a literatura respaldam a introdução de um programa de vigilância no nosso hospital.

\section{Bibliografía}

1. Graham HK, Rosenbaum P, Paneth N, Dan B, Lin JP, Damiano DL, et al. Cerebral palsy. Nat Rev Dis Primers 2016; 2:15082.

2. Hägglund G, Andersson S, Düppe H, Lauge-Pedersen H, Nordmark E, Westbom L. Prevention of hip dislocation in children with cerebral palsy. The first ten years experiences of a population-based prevention programme. J Bone Joint Surg Br 2005; 87(1):95-101.

3. Pruszczynski B, Sees J, Miller F. Risk factors for hip displacement in children with cerebral palsy: systematic review. J Pediatr Orthop 2016; 36(8):829-33.

4. Reimers $\mathbf{J}$. The stability of the hip in children. A radiological study of the results of muscle surgery in cerebral palsy. Acta Orthop Scandin Suppl 1980; 184:1-100.

5. Hermanson M, Hägglund G, Riad J, Rodby-Bousquet $\mathbf{E}$, Wagner P. Prediction of hip displacement in children with cerebral palsy. Bone Joint J 2015; 97-B(10):1441-4.

6. Hermanson $\mathbf{M}$, Hägglund $\mathbf{G}$, Riad $\mathbf{J}$, Wagner $\mathbf{P}$. Head-shaft angle is a risk factor for hip displacement in children with cerebral palsy. Acta Orthop 2015; 86(2):229-32.

7. Givon U. Management of the spactic hip in cerebral palsy. Curr Opin Pediatr 2017; 29(1):65-9. 
8. Hägglund G, Alriksson-Schmidt A, Lauge-Pedersen H, Rodby-Bousquet E, Wagner P, Wastbom L. Prevention of dislocation of the hip in children with cerebral palsy: 20-year results of a population-based prevention programme. Bone Joint J 2014; 96-B(11):1546-52.

9. Elkamil Al, Andersen GL, Hägglund G, Lamvik T, Skranes J, Vik T. Prevalence of hip dislocation among children with cerebral palsy in regions with and without a surveillance programme: a cross sectional study in Sweden and Norway. BMC Musculoskel Dis 2011; 12:284

10. Kentish M, Wybter M, Snape N, Boyd R. Five-year outcome of state-wide hip surveillance of children and adolescents with cerebral palsy. J Pediatr Rehabil Med 2011; 4(3):20517.

11. Hägglund G. Radiographic follow-up in CPUP to prevent hip dislocation. 2013. Disponible en: http://cpup.se/wp-content/uploads/2013/07/CPUPprevent_hip_dislocation20130210.pdf [Consulta: 24 setiembre 2018].

12. Rehbein I. Análisis de los procedimientos quirúrgicos ortopédicos realizados en niños con parálisis cerebral en relación con la edad y función motora gruesa. [Monografía Postgrado] Montevideo: Traumatología y Ortopedia, Facultad de Medicina-UdelaR, 2018:24 p.

13. Palisano R, Rosenbaum P, Walter S, Russell D, Wood E, Galuppi B. Development and reliability of a system to classify gross motor function in children with cerebral palsy. Dev Med Child Neurol 1997; 39(4):214-23.

14. Gainsborough M, Surman G, Maestri G, Colver A, Cans C. Validity and reliability of the guidelines of the surveillance of cerebral palsy in Europe for the classification of cerebral palsy. Dev Med Child Neurol 2008; 50(11):828-31.

15. Hägglund G, Lauge-Pedersen $\mathbf{H}$, Wagner $\mathbf{P}$. Characteristics of children with hip displacement in cerebral palsy. BMC Musculoskeletal Disord 2007; 8:101.

16. Hägglund G, Lauge-Pedersen H, Persson M. Radiographic threshold values for hip screening in cerebral palsy. J Child Orthop 2007(1):43-7.

17. AppInConf AB. CPUP Hip Score [APP]. Lund, Suecia AppInConf AB, 2019. Disponible para IPhone y Android.

18. Southwick WO. Osteotomy through the lesser trochanter for slipped capital femoral epiphysis. J Bone Joint Surg Am 1967; 49(5):807-35.

19. Hermanson M, Hägglund G, Riad J, Rodby-Bousquet E. Inter-and intra-rater reliability of the head-shaft angle in children with cerebral palsy. J Child Orthop 2017; 11(4):256-62.

20. Craven A, Pym A, Boyd RN. Reliability of radiologic measures of hip displacement in a cohort of preschool-aged children with cerebral palsy. J Pediatr Orthop 2014; 34(6): 597-602.

21. Faraj S, Atherton WG, Stott NS. Inter- and intra-measurer error in the measurement of Reimer's hip migration percentage. J Bone Joint Surg Br 2004; 86(3):434-7.

22. Gordon GS, Simkiss DE. A systematic review of the evidence for hip surveillance in children with cerebral palsy. J Bone Joint Surg Br 2006; 88(11):1492-6.

23. Wynter M, Gibson N, Kentish M, Love S, Thomason P, Kerr Graham H. The consensus statement on hip surveillance for children with cerebral palsy: australian standards of care. J Pediatr Rehabil Med 2011; 4(3):183-95.

\section{Contribución de autores}

Viviana Teske, https://orcid.org/0000-0003-4407-6501. Asistió con aportes sobre la concepción y diseño del estudio, desarrollo de protocolos de estudio, y fue responsable de identificar las historias clínicas de los pacientes y de recopilar datos demográficos, asistió con el análisis e interpretación de los datos y la redacción, edición y revisión de manuscritos. Johan von Heideken, https://orcid.org/0000-0003-3604-9167. responsable de la concepción y el diseño del estudio, el desarrollo de los protocolos del estudio, la asistencia en el análisis y la interpretación de los datos y participó en la redacción, edición y revisión de manuscritos.

Ignacio Rehbein, https://orcid.org/0000-0002-1647-4935. Asistió con aportes sobre la concepción y el diseño del estudio, el desarrollo de protocolos de estudio y fue responsable de la recopilación de datos demográficos, el análisis e interpretación de los datos y radiografías y la redacción y edición de manuscritos. 